\title{
IAMJ
}

INTERNATIONAL

AYURVEDIC

MEDICAL JOURNAL

\section{A COMPARATIVE CLINICAL STUDY OF SIDHARTAK LEPA, VAMAN \& VIRECHAN IN THE MANAGEMENT OF “EKAKUSTHA” W.S.R. TO PSORIASIS}

\author{
Richa Parmar', Mahesh Kumar Sharma², Gyan Prakash Sharma ${ }^{3}$ \\ ${ }^{1}$ P. G. Scholar of Panchakarma Dept., UCA, DSRRAU, Jodhpur, Rajasthan, India \\ ${ }^{2}$ M.D. (Ayu.), Associate Professor \& H.O.D., P.G. Department of Panchakarma, UCA, DSRRAU, Jodhpur, \\ Rajasthan, India \\ ${ }^{3}$ M.D. (Panchakarma), Assistant Professor, UCA, DSRRAU, Jodhpur, Rajasthan, India
}

Corresponding Author: parmaricha90@gmail.com

\section{https://doi.org/ $10.46607 /$ iamj0108122020}

(Published online: December 2020)

Open Access

(C) International Ayurvedic Medical Journal, India 2020

Article Received: 12/10/2020 - Peer Reviewed: 04/11/2020 - Accepted for Publication: 08/11/2020

\section{(A) Check for updates}

\section{ABSTRACT}

Psoriasis is a chronic autoimmune condition that triggers the buildup of skin cells too quickly. This buildup of cells formed scaling to the surface of the skin. It became basically Inflammation and redness across the scales. Typical colour of psoriatic scales is whitish \& silver, which occurs on the skin in dense red patches. Those patches can crack and bleed at times. (Dry Erythematous, Macules, Papules \& Scales). Psoriasis is one the worst dermatological disorder in the world affects up to $3.5 \%$ and the Indian population roughly 0.44 to $2-8 \%$. All the skin diseases in Ayurveda have been described under the broad heading of "Kustha Roga". Kustha divided in 2 types: - Maha Kustha \& Ksudra Kustha. Ekakustha is considered one of the Kshudra Kustha. Ayurvedic Samhitas have considered all type of Kustha is Tridosaja. On the basis of dominance of Dosha they have different types. So that Ekakustha is (Vata-Kaphaj) phenomenon. Ekakustha is (Vata-Kaphaja) phenomenon. Psoriasis has a wide range of presentation from just a single spot to involvement of all over the body. When we carefully analyses the nature, we can see close resemblance of symptoms between Psoriasis \& Ekakustha. This Comparative Clinical study shows that combined three procedures-Vaman Karma, Virechana Karma \& Lepa by Sidhartak Churna is showing more effective results in reliving Psoriasis in comparison of single procedure like Vaman Karma by Sidhartak 
Churna, Virechan Karma by Sidhartak Churna \& Sidhartak Lepa is showing less relief than combined 3 procedure.

Keywords: Psoriasis, Ekakustha, Sidharthak Churna.

\section{INTRODUCTION}

The largest organ of body is called Skin. It prevents germs from getting into the body and save internal organs. Skin protects the survival of all other parts of the body and even maintains the immune system. Skin represents human personality so everyone cares for their skin. Any skin disease can affect people's physical and mental health. Skin is just like a mirror showing our emotions and natural physiological aspects. Patient of skin disorder feel depressed and low confidence in social life. Psoriasis is one the worst dermatological disorder in the world affects up to $3.5 \%$ and the Indian population roughly 0.44 to $2-8 \%{ }^{1}$ All of Ayurveda's skin diseases were listed under the big heading "Kustha Roga", the disease that destroy the look and personality is called Kustha Roga. ${ }^{2}$ The present study of psoriasis was taken as Ekakustha. The Ekakustha phenomenon is Vata-Kaphaja. Psoriasis has a wide range of manifestations, from just one spot to affecting the whole body. When we examine the existence carefully, we can see strong symptom similarity between Psoriasis \& Ekakustha, So Psoriasis has been taken as Ekakustha in present research. In present study we selected Vaman \& Virechan with Sidharthak Churna. ${ }^{3}$

\section{Need of Study-}

- There is a great need for affordable \& inexpensive as well as safe $\&$ efficient new treatment methods.

- In such cases Ayurvedic medicines are useful.

- To Compare the Single procedure effect with three combined procedure effect in Psoriasis, and effect of Sidhartak Churna in psoriasis.

\section{Aim \& Objectives-}

- To Analyse the effect of "Vaman Karma" as Shodhana Karma in the management of Ekakustha.

- To Analyse the effect of "Virechan Karma” Shodhana Karma in the management of Ekakustha.

- To Analyses the effect of "Sidhartak Lepa" External medicine in the management of Ekakustha
- To compare the effect of "Vaman Karma \& Virechan Karma" both Shodhana Karma and Sidhartak Lepa on Ekakustha.

\section{Material \& Methods:}

\section{A. Selection of patient $\&$ Criteria of diagnosis:}

The study was conducted on 40 clinically diagnosed $\&$ confirmed cases of Psoriasis from OPD \& IPD section DSRRAU, Jodhpur. The main diagnosis criteria for patients were based on the Ayurveda based cardinal signs and symptoms of the disease and modern texts.

\section{B. Criteria of Inclusion:}

1. Diagnosed \& confirmed cases of Ekakustha (Psoriasis), on the basis of sign \& symptoms.

2. Patients between the age group of 18-60 years of either sex.

C. Exclusion criteria:

1. Patients below $18 \mathrm{yrs}$ and above 60yrs.

2. Infective origin, Allergic diseases, Immune compromised like HIV, HBV.

3. Pregnant and lactating woman.

4. Patient having cardiac disease, renal disease and endocrine disorder will exclude from study to avoid overlapping of symptomatology.

\section{Investigations:}

1. ESR (erythrocyte sedimentation rate) 2. TLC, 3. Blood sugar (Fasting/ Post Prandial) 4.Sr. Cholesterol, 5. HB $\%, 6$. Sr. Triglyceride

\section{E. Grouping:}

40 registered patients were randomly divided into four groups named as group A, B, C \& D consisting 10 patients each.

Group A: 10 registered patients of Psoriasis administered with "Vaman Karma" with "Sidhartak Churna".

Group B: 10 registered patients of Psoriasis administered with "Virechan Karma" with "Sidhartak Churna." 
Group C: 10 registered patients of Psoriasis administered "Lepa" with "Sidhartak Churna".

Group D: 10 registered patients of Psoriasis administered "Vaman Karma", "Virechan Karma" \& "Lepa" with "Sidhartak Churna".

\section{F. Procedure Protocol:}

1. Vaman Karma: Procedure of Vaman was carried out as follows, Deepana-Pachana with Panchkol Churna 3gm BD for three days, Snehapana was done with Go Ghrita till Samyak Sneha lakshana was observed, and then Sarvang Snehana Swedana was done. Vaman was induced by decoction $180 \mathrm{ml}$ Sidhartak Churna (Madanaphala double quantity), Saindhava Namaka 1 gm \& honey $30 \mathrm{gm}$ as Vamaka Yoga after that Dhoompana \& Samsarjana Karma was carried out.

2. Virechan Karma: Deepana- Pachana with Panchkol churna $3 \mathrm{gm} \mathrm{BD}$ for 3 days, after it Snehapana was done by Go Ghrita for three days.
Then Sarvang Snehana Swedana was done for three days \& Virechan with decoction of Sidhartak Churna $80 \mathrm{ml}$ (Aragwadha in 2double quantity) was given empty stomach and after that proper Samsarjana Karma was done.

3. Lepa with Sidhartak Churna: Apply Sidhartak Lepa for 15 days.

4. Vaman Karma, Virechna Karma \& Lepa with Sidhartak Churna:

G. Duration of clinical trial and follow up: Total duration of trial was 2 months with follow up after every 15 days and after completion of trial.

\section{H. Termination criteria:}

1. Progressive worsening of Psoriasis \& development of complication during trial period.

2. Patient who does not complete the treatment with follow-up.

3. Death of patient due to any cause.

\section{Criteria of assessment:}

\section{Subjective Parameters-}

1. Aswedanam: (Lesions are dry rough)

\begin{tabular}{|l|l|}
\hline No dryness and roughness & 0 \\
\hline Slite dryness and roughness & 1 \\
\hline One or more than two lesions & 2 \\
\hline All over the body & 3 \\
\hline
\end{tabular}

2. Mahavastum: (Bigger lesions are found all over the body and deeply rooted)

Not Present

One or more than two lesions

0

Two or more bigger lesion over more than one body part

Lesion all over the body with silvery scales

3. Matsyashakalopamam: (Well defined raised macules, papules, and erythematic plaques found which covered with silvery scales)

No

Up to $25 \%$

26 to $50 \%$

More than 50\%

0
1
2
3

4. Krishna Arun Varna: (Lesions are raised and erythematous, thick indurated, lesion becomes black in colour)

\begin{tabular}{|l|l|}
\hline Normal skin & 0 \\
\hline Slite black & 1 \\
\hline Dark patches & 2 \\
\hline Black spots with variation & 3 \\
\hline
\end{tabular}


Severity of each variable will range from; these criteria will be assessed and scored as follows -

\begin{tabular}{|l|l|l|}
\hline 0 & - & Normal \\
\hline 1 & - & Mild \\
\hline 2 & - & Moderate \\
\hline 3 & - & Severe \\
\hline
\end{tabular}

PASI Score: PASI $=$ Total Head + Total Arms + Total Trunk + Total Legs

Interpretation: Minimum Score $=0$; Maximum Score $=72$

OBSERVATION: The present study showed that relative preponderance of the incidence of Psoriasis is in 2nd to 4th decade of life of Vata- Kaphaja Prakriti males \& Hindu dominated families, socioeconomic status middle class, married persons were found to suffer more, runs chronic course, maximum number of trial patients were govt. employs, more in vegetarian subjects \& no significant family history, maximum affected urban population having graduate education group suffered mostly from disease. Subjects addicted to Tea, Tobacco \& Alcohol which are pathologic risk factor for Psoriasis, Chinta (stress) found in maximum patients who lead to Depression- psychological stress as being a key exacerbation or trigger of Psoriasis. Several etiological factors as described in Ayurveda and Modern medicine for initiation of Ekakustha (Psoriasis) were found to be responsible as precipitating factors in current clinical study.

Results: Table is showing effect of therapeutic trial on clinical symptomatology in 40 patients of Ekakustha (Psoriasis) based on intra group comparison (Wilcoxon matched pairs signed rank test)

Table 5

\begin{tabular}{|c|c|c|c|c|c|c|c|c|c|}
\hline \multirow[t]{2}{*}{ Variable } & \multirow[t]{2}{*}{ Gr. } & \multicolumn{2}{|l|}{ Mean } & \multirow[t]{2}{*}{ Diff. } & \multirow[t]{2}{*}{$\%$ Change } & \multirow[t]{2}{*}{$\mathbf{S D} \pm$} & \multirow[t]{2}{*}{$\mathbf{S E} \pm$} & \multirow[t]{2}{*}{$\mathbf{P}$} & \multirow[t]{2}{*}{$\mathbf{S}$} \\
\hline & & BT & AT & & & & & & \\
\hline \multirow[t]{4}{*}{ Aswedanam } & A & 2.100 & 1.000 & 1.100 & $52.38 \%$ & 0.3162 & 0.1000 & 0.0002 & E.S \\
\hline & B & 2.300 & 1.200 & 1.100 & $47.82 \%$ & 0.3162 & 0.1000 & 0.0011 & V.S \\
\hline & C & 1.900 & 1.100 & 0.8000 & $42.10 \%$ & 0.4216 & 0.1333 & 0.0039 & V.S \\
\hline & $\mathrm{D}$ & 2.400 & 1.000 & 1.400 & $58.33 \%$ & 0.5164 & 0.1633 & 0.0010 & E.S \\
\hline \multirow[t]{4}{*}{ Mahavastum } & A & 1.900 & 0.900 & 1.000 & $52.63 \%$ & 0.4714 & 0.1491 & 0.0020 & V.S \\
\hline & B & 2.200 & 1.100 & 1.100 & $50 \%$ & 0.5676 & 0.1795 & 0.0020 & V.S \\
\hline & $\mathrm{C}$ & 1.800 & 1.000 & 0.8000 & $44.44 \%$ & 0.4216 & 0.1333 & 0.0039 & V.S \\
\hline & $\mathrm{D}$ & 2.200 & 1.100 & 1.200 & $54.54 \%$ & 0.4216 & 0.1333 & 0.0010 & E.S \\
\hline \multirow[t]{4}{*}{ Matsyashkalopamam } & A & 2.000 & 1.000 & 1.000 & $50 \%$ & 0.4714 & 0.1491 & 0.0020 & V.S \\
\hline & B & 2.300 & 1.200 & 1.100 & $47.82 \%$ & 0.3162 & 0.1000 & 0.0010 & E.S \\
\hline & $\mathrm{C}$ & 1.900 & 1.000 & 0.9000 & $47.36 \%$ & 0.5676 & 0.1795 & 0.0039 & V.S \\
\hline & $\mathrm{D}$ & 2.100 & 1.000 & 1.100 & $52.38 \%$ & 0.5676 & 0.1795 & 0.0020 & V.S \\
\hline \multirow[t]{4}{*}{ Krishna Arun Varna } & A & 2.000 & 0.900 & 1.100 & $55 \%$ & 0.3162 & 0.1000 & 0.0010 & E.S \\
\hline & B & 1.900 & 0.900 & 1.000 & $52.63 \%$ & 0.4714 & 0.1491 & 0.0020 & V.S \\
\hline & $\mathrm{C}$ & 1.600 & 0.800 & 0.8000 & $50 \%$ & 0.4216 & 0.1333 & 0.0039 & V.S \\
\hline & $\mathrm{D}$ & 2.300 & 1.000 & 1.300 & $56.52 \%$ & 0.4830 & 0.1528 & 0.0010 & E.S \\
\hline \multirow[t]{4}{*}{ PASI Score } & A & 25.170 & 9.090 & 16.080 & $63.88 \%$ & 3.788 & 1.198 & 0.0010 & E.S \\
\hline & B & 24.900 & 9.430 & 15.470 & $62.12 \%$ & 3.071 & 0.9710 & 0.0010 & E.S \\
\hline & $\mathrm{C}$ & 18.460 & 8.550 & 9.910 & $53.68 \%$ & 2.799 & 0.8850 & 0.0010 & E.S \\
\hline & $\mathrm{D}$ & 27.630 & 9.000 & 18.630 & $67.42 \%$ & 2.732 & 0.8640 & 0.0010 & E.S \\
\hline
\end{tabular}


Table 6: Table is showing effect of therapeutic trial on clinical symptomatology in 40 patients of Ekakustha (Psoriasis) based on INTER GROUP comparison- Kruskal Wallis test (non-parametric ANOVA)

\begin{tabular}{|c|c|c|c|c|}
\hline Parameter & $P$ value & Significant & Kruskal wallis static & K.W. test \\
\hline Aswedanam & 0.0275 & $\mathrm{~S}$ & 9.137 & $\begin{array}{ll}\text { A Vs B } & \text { N.S } \\
\text { A Vs C } & \text { N.S } \\
\text { A Vs D } & \text { N.S } \\
\text { B Vs C } & \text { N.S } \\
\text { B Vs D } & \text { N.S } \\
\text { C Vs D } & \text { S }\end{array}$ \\
\hline Mahavastum & 0.2719 & N.S & 3.905 & $\begin{array}{ll}\text { A Vs B } & \text { N.S } \\
\text { A Vs C } & \text { N.S } \\
\text { A Vs D } & \text { N.S } \\
\text { B Vs C } & \text { N.S } \\
\text { B Vs D } & \text { N.S } \\
\text { C Vs D } & \text { N.S }\end{array}$ \\
\hline Matsyashakalopamam & 0.6339 & N.S & 1.714 & $\begin{array}{ll}\text { A Vs B } & \text { N.S } \\
\text { A Vs C } & \text { N.S } \\
\text { A Vs D } & \text { N.S } \\
\text { B Vs C } & \text { N.S } \\
\text { B Vs D } & \text { N.S } \\
\text { C Vs D } & \text { N.S }\end{array}$ \\
\hline Krishna Arun Varna & 0.0938 & $\mathrm{~S}$ & 6.397 & $\begin{array}{ll}\text { A Vs B } & \text { N.S } \\
\text { A Vs C } & \text { N.S } \\
\text { A Vs D } & \text { N.S } \\
\text { B Vs C } & \text { N.S } \\
\text { B Vs D } & \text { N.S } \\
\text { C Vs D } & \text { N.S }\end{array}$ \\
\hline PASI Score & 0.0002 & E.S & 19.976 & $\begin{array}{ll}\text { A Vs B } & \text { N.S } \\
\text { A Vs C } & \text { S } \\
\text { A Vs D } & \text { N.S } \\
\text { B Vs C } & \text { N.S } \\
\text { B Vs D } & \text { N.S } \\
\text { C Vs D } & \text { E.S }\end{array}$ \\
\hline
\end{tabular}

Table 7: Table is showing effect of therapeutic trial on Laboratory parameters in patients of Ekakustha (Psoriasis) based on intra group comparison (Pair t-test)

\begin{tabular}{|c|c|c|c|c|c|c|c|c|c|}
\hline \multirow[t]{2}{*}{ Variable } & \multirow[t]{2}{*}{ Gr. } & \multicolumn{2}{|l|}{ Mean } & \multirow[t]{2}{*}{ Diff. } & \multirow[t]{2}{*}{ SD \pm} & \multirow[t]{2}{*}{$\mathbf{S E} \pm$} & \multirow[t]{2}{*}{$\mathbf{P}$} & \multirow[t]{2}{*}{$\mathbf{T}$} & \multirow[t]{2}{*}{$\mathbf{S}$} \\
\hline & & BT & AT & & & & & & \\
\hline \multirow[t]{4}{*}{$\mathrm{HB} \%$} & A & 13.830 & 14.670 & -0.840 & 1.007 & 0.3184 & 0.0135 & 2.638 & $\mathrm{~S}$ \\
\hline & B & 13.450 & 13.910 & -0.460 & 0.4719 & 0.1492 & 0.0065 & 3.083 & V.S \\
\hline & $\mathrm{C}$ & 13.060 & 13.750 & -0.690 & 0.585 & 0.1853 & 0.0024 & 3.724 & V.S \\
\hline & D & 13.920 & 14.620 & -0.700 & 0.7674 & 0.2427 & 0.0090 & 2.885 & V.S \\
\hline \multirow[t]{4}{*}{ TLC } & A & 6220.0 & 6045.0 & 175.00 & 773.61 & 244.64 & 0.2463 & 0.7153 & N.S \\
\hline & B & 6270.0 & 6085.0 & 185.00 & 537.51 & 169.98 & 0.1524 & 1.088 & N.S \\
\hline & $\mathrm{C}$ & 5720.0 & 5520.0 & 200.0 & 588.78 & 186.19 & 0.1553 & 1.074 & N.S \\
\hline & D & 5940.0 & 5595.0 & 345.0 & 453.66 & 143.46 & 0.0198 & 2.405 & $\mathrm{~S}$ \\
\hline ESR & A & 17.700 & 14.300 & 3.400 & 1.430 & 0.4522 & $<0.0001$ & 7.520 & E.S \\
\hline
\end{tabular}


Richa Parmar et al: A Comparative Clinical Study of Sidhartak Lepa, Vaman \& Virechan in the Management of "Ekakustha" w.s.r. to Psoriasis

\begin{tabular}{|c|c|c|c|c|c|c|c|c|c|}
\hline & B & 18.400 & 15.000 & 3.400 & 1.075 & 0.3399 & $<0.0001$ & 10.002 & E.S \\
\hline & $\mathrm{C}$ & 19.300 & 17.500 & 1.800 & 3.521 & 1.114 & 0.0702 & 1.616 & N.S \\
\hline & $\mathrm{D}$ & 20.200 & 16.900 & 3.300 & 1.494 & 0.4726 & $<0.0001$ & 6.983 & E.S \\
\hline \multirow[t]{4}{*}{ Sr. Cholesterol } & A & 194.60 & 169.20 & 25.400 & 9.336 & 2.952 & $<0.0001$ & 8.604 & E.S \\
\hline & B & 206.40 & 173.60 & 32.800 & 23.098 & 7.304 & 0.0008 & 4.491 & E.S \\
\hline & $\mathrm{C}$ & 204.70 & 202.90 & 1.800 & 16.745 & 5.295 & 0.3709 & 0.3399 & N.S \\
\hline & $\mathrm{D}$ & 185.00 & 156.40 & 28.600 & 9.143 & 2.891 & $<0.0001$ & 9.892 & E.S \\
\hline \multirow[t]{4}{*}{ Sr. Triglyceride } & A & 124.60 & 98.900 & 25.700 & 10.771 & 3.406 & $<0.0001$ & 7.545 & E.S \\
\hline & B & 132.40 & 100.60 & 31.800 & 11.173 & 3.533 & $<0.0001$ & 9.000 & E.S \\
\hline & $\mathrm{C}$ & 114.60 & 106.10 & 8.500 & 14,316 & 4.527 & 0.0466 & 1.878 & $\mathrm{~S}$ \\
\hline & $\mathrm{D}$ & 107.60 & 89.400 & 18.200 & 7.969 & 2.520 & $<0.0001$ & 7.222 & E.S \\
\hline \multirow[t]{4}{*}{ Blood Sugar (Fasting) } & A & 97.140 & 92.330 & 4.810 & 8.875 & 2.806 & 0.0603 & 1.714 & N.S \\
\hline & B & 97.830 & 92.050 & 5.780 & 6.344 & 2.006 & 0.0091 & 2.881 & V.S \\
\hline & $\mathrm{C}$ & 91.170 & 88.400 & 2.770 & 4.673 & 1.478 & 0.0468 & 1.874 & $\mathrm{~S}$ \\
\hline & $\mathrm{D}$ & 97.780 & 96.800 & 0.9800 & 7.189 & 2.273 & 0.3383 & 0.4311 & N.S \\
\hline
\end{tabular}

Table 8: Table is showing effect of therapeutic trial on Laboratory parameters in 40 patients of Ekakustha (Psoriasis) based on INTER GROUP comparison (ANOVA- Tukey Kramer multiple comparison test)

\begin{tabular}{|c|c|c|c|}
\hline Parameter & P Value & Significant & Tukey-Kramer test \\
\hline $\mathrm{HB} \%$ & 0.7142 & N.S & $\begin{array}{ll}\text { A Vs B } & \text { N.S } \\
\text { A Vs C } & \text { N.S } \\
\text { A Vs D } & \text { N.S } \\
\text { B Vs C } & \text { N.S } \\
\text { B Vs D } & \text { N.S } \\
\text { C Vs D } & \text { N.S }\end{array}$ \\
\hline TLC & 0.9113 & N.S & $\begin{array}{ll}\text { A Vs B } & \text { N.S } \\
\text { A Vs C } & \text { N.S } \\
\text { A Vs D } & \text { N.S } \\
\text { B Vs C } & \text { N.S } \\
\text { B Vs D } & \text { N.S } \\
\text { C Vs D } & \text { N.S }\end{array}$ \\
\hline ESR & 0.2641 & N.S & $\begin{array}{ll}\text { A Vs B } & \text { N.S } \\
\text { A Vs C } & \text { N.S } \\
\text { A Vs D } & \text { N.S } \\
\text { B Vs C } & \text { N.S } \\
\text { B Vs D } & \text { N.S } \\
\text { C Vs D } & \text { N.S }\end{array}$ \\
\hline Sr. Cholesterol & 0.0004 & E.S & $\begin{array}{ll}\text { A Vs B } & \text { N.S } \\
\text { A Vs C } & \text { S } \\
\text { A Vs D } & \text { N.S } \\
\text { B Vs C } & \text { E.S } \\
\text { B Vs D } & \text { N.S } \\
\text { C Vs D } & \text { V.S }\end{array}$ \\
\hline Sr. Triglyceride & 0.0003 & E.S & $\begin{array}{ll}\text { A Vs B } & \text { N.S } \\
\text { A Vs C } & \text { V.S } \\
\text { A Vs D } & \text { N.S } \\
\text { B Vs C } & \text { E.S }\end{array}$ \\
\hline
\end{tabular}




\begin{tabular}{|c|c|c|c|}
\hline & & & $\begin{array}{ll}\text { B Vs D } & \text { N.S } \\
\text { C Vs D } & \text { N.S }\end{array}$ \\
\hline Blood Sugar (Fasting) & 0.4251 & N.S & $\begin{array}{ll}\text { A Vs B } & \text { N.S } \\
\text { A Vs C } & \text { N.S } \\
\text { A Vs D } & \text { N.S } \\
\text { B Vs C } & \text { N.S } \\
\text { B Vs D } & \text { N.S } \\
\text { C Vs D } & \text { N.S }\end{array}$ \\
\hline
\end{tabular}

\section{Overall Effect of Clinical Trial:}

Table 9: Table is showing the criteria for the assessment of overall effect of the therapies:

\begin{tabular}{|l|l|l|l|}
\hline S. No. & Symptoms Relieved & Grading & Assessment \\
\hline 1 & Less than $25 \%$ & Mild relief & Non-satisfactory \\
\hline 2 & $25 \%$ to $50 \%$ & Moderate relief & Good \\
\hline 3 & $50 \%$ to $75 \%$ & Significant relief & Satisfactory \\
\hline 4 & $75 \%$ to $100 \%$ & Complete relief & Excellent \\
\hline
\end{tabular}

Table 10: Table is showing the \% Relief of Subjective parameters in the Group A, B, C \& D

\begin{tabular}{|l|l|l|l|l|}
\hline Subjective parameters & \% Relief in Group A & \% Relief in Group B & \% Relief in Group C & \% Relief in Group D \\
\hline Aswedanam & $52.38 \%$ & $47.82 \%$ & $42.10 \%$ & $58.33 \%$ \\
\hline Mahavastum & $52.63 \%$ & $50 \%$ & $44.44 \%$ & $54.54 \%$ \\
\hline Matsyashakalopamam & $50 \%$ & $47.82 \%$ & $47.36 \%$ & $52.38 \%$ \\
\hline Krishna Arun Varna & $55 \%$ & $52.63 \%$ & $50 \%$ & $56.52 \%$ \\
\hline PASI Score & $63.88 \%$ & $62.12 \%$ & $53.68 \%$ & $67.42 \%$ \\
\hline Total & $54.77 \%$ & $52.07 \%$ & $47.51 \%$ & $57.83 \%$ \\
\hline
\end{tabular}

Table 11: Table is showing the \% Relief of Objective parameters in the Group A, B, C \& D

\begin{tabular}{|l|l|l|l|l|}
\hline Objective parameters & \% Relief in Group A & \% Relief in Group B & \% Relief in Group C & \% Relief in Group D \\
\hline HB\% & $6.07 \%$ & $3.42 \%$ & $5.28 \%$ & $5.02 \%$ \\
\hline TLC & $2.81 \%$ & $2.95 \%$ & $3.49 \%$ & $5.80 \%$ \\
\hline ESR & $19.20 \%$ & $18.47 \%$ & $9.32 \%$ & $16.33 \%$ \\
\hline Sr.Cholesterol & $13.05 \%$ & $15.89 \%$ & $0.87 \%$ & $15.45 \%$ \\
\hline Sr.Triglyceride & $20.62 \%$ & $24.01 \%$ & $7.41 \%$ & $16.91 \%$ \\
\hline Blood Sugar (Fasting) & $4.95 \%$ & $5.90 \%$ & $3.03 \%$ & $1.00 \%$ \\
\hline
\end{tabular}

\section{DISCUSSION}

Discussion on Clinical Study: The patients suffering from skin disease Psoriasis (Eka-Kustha) attending the O.P.D./I.P.D. section of P.G. Department of Panchkarma, DSRRAU, Jodhpur (Raj.), were randomly selected who fulfilling the inclusion and exclusion criteria of study. Total 40 patients were registered for the current trial and completed the therapy. The drug for trial Sidharthak Churna was chosen from Charaka Samhita Chikitsa Sthan - Kustha Roga Chikitsa contents of the Sidharthak Churna are Nagaramotha, Madanaphala, Amalaki, Vibhitaki, Hareetaki, Karan- ja, Aragwadha, Kalinga Yava, Daruharidra, Saptaparna in equal proportion for Sidharthak Churna, Madanaphala in 2 part for Vaman Karma, Aragwadha in 2 part for Virechan Karma.

Intra Group Comparison: Comparison between Group A, B, C \& D for Subjective parameters, Group A (The medicine Group) showed statistically Extremely significant result in Aswedanam, Very Significant result in Mahavastum, Matsyashakalopamam, Extremely Significant result in Krishna Arun Varna, Extremely significant result in PASI Score. Group B (The medicine Group) showed statistically Very significant result in Aswedanam, Mahavastum, Krishna 
Arun Varna Extremely Significant result in Matsyashakalopamam PASI Score. Group C (The medicine Group) showed statistically Very significant result in Aswedanam, Mahavastum, Matsyashakalopamam, Krishna Arun Varna Extremely Significant result in PASI Score.

Group D (The medicine Group) showed statistically extremely significant result in Aswedanam, V Mahavastum, Krishna Arun Varna, PASI Score, and Very significant Matsyashakalopamam. Comparison between Group A, B, C \& D for Objective parameters: In patients of Group A showed statistically Significant result in HB\%, Not Significant result in TLC, Blood Sugar (Fasting) Extremely Significant result in ESR, Sr. Cholesterol, Sr. Triglyceride.

\section{CONCLUSION}

Result reveled that symptomatically $54.77 \%$ relief was observed in Group A while 52.07\% relief was observed in Group B, 47.51\% relief was observed in Group C and 57.83\% relief was observed in Group D.
Comparing the symptomatic improvement in all Four groups it was found that average percentage of relief was higher in Group D (Vaman Karma, Virechan Karma \& Sidhartak Lepa). Therefore, it can be concluded that Shodhana (Vaman \& Virechan) By Sidhartak Churna \& Sidhartak Lepa administration are effective in management of Eka-Kushtha (Psoriasis) as these are safe, cost effective and free from any side effects.

\section{REFERENCES}

1. Kurd SK, Gelfand JM. The prevalence of previously diagnosed and undiagnosed psoriasis in US adults: results from NHANES 2003-2004. J AM Acad Dermatol.2009; 60(2):218-24.)

2. Charaka Samhita, Part 2nd of Agnivesa Revised by Caraka \& Dradhabala by Pt. Kasinatha Sastri, Dr. Gorakha Nath Chaturvedi, Chokhamba Bharati Academy, Varanasi, Reprint Year: 2009 Ch. Chi. 7/2

3. Charaka Samhita, Part 2nd of Agnivesa Revised by Caraka \& Dradhabala by Pt. Kasinatha Sastri, Dr. Gorakha Nath Chaturvedi, Chokhamba Bharati Academy, Varanasi, Reprint Year: 2009 Ch. Chi.7/12

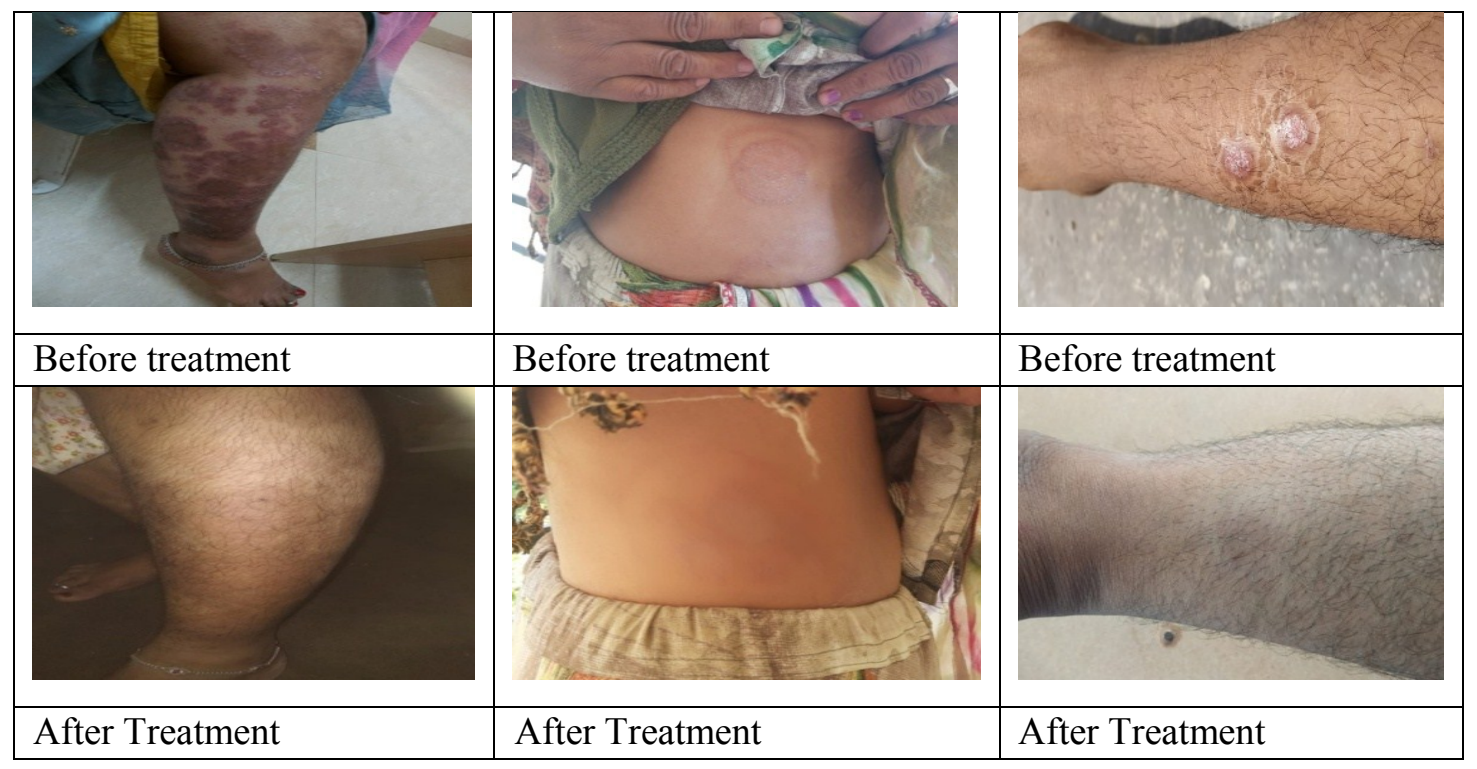

\section{Source of Support: Nil Conflict of Interest: None Declared}

How to cite this URL: Richa Parmar et al: A Comparative Clinical Study of Sidhartak Lepa, Vaman \& Virechan in the Management of "Ekakustha" w.s.r. to Psoriasis. International Ayurvedic Medical Journal \{online\} 2020 \{cited December, 2020\} Available from: http://www.iamj.in/posts/images/upload/5199_5206.pdf 